positivity and use of GCs. Oral and ocular damage items significantly correlated with ESSPRI, OHIP and OSDI, whereas systemic damage items positively correlated with patients $\mathrm{CCl}$.

Conclusions: This large pSS cohort confirmed that demographic and clinical characteristics as well as medication are independently associated with diseaserelated and treatment-related damage. In particular, this study shows a highly significant impact of baseline disease activity on the development of future damage and poor PROs in pSS patients.

Disclosure of Interest: None declared

DOI: 10.1136/annrheumdis-2018-eular.5797

\section{FRI0353 DEVELOPMENT AND VALIDATION OF QUESTIONNAIRES TO ASSESS HEALTHCARE UTILISATION AND ACCESS IN COHORTS OF PATIENTS WITH PRIMARY SJOÖGREN'S SYNDROMEAT THE DIAGNOSIS AND DURING THE DISEASE COURSE}

C. Seghieri ${ }^{1}$, T. Grillo Ruggieri ${ }^{1}$, C. Baldini ${ }^{2}$, L. Quartuccio ${ }^{3}$, R. Priori ${ }^{4}$, E. Bartoloni ${ }^{5}$ F. Carubbi ${ }^{6}$, M. Orlandi ${ }^{7}$, S. Gandolfo ${ }^{3}$, S. De Vita ${ }^{3}$, S. Bombardieri ${ }^{2}$ on behalf of Italian Rheumatology Society-pSS study group and of the HarmonicSS "Harmonization and integrative analysis of regional, national and international Cohorts on pSS towards improved stratification, treatment and health policy making" group. 'Sant'Anna School of Advanced Studies; ${ }^{2}$ University of Pisa, Pisa; ${ }^{3}$ University of Udine, Udine; ${ }^{4}$ University of Rome, Rome; ${ }^{5}$ University of Perugia, Perugia; ${ }^{6}$ University of L'Aquila, L'Aquila; ${ }^{7}$ University of Florence, Florence, Italy

Background: The geographic variation in healthcare spending, utilisation and quality, across and within countries is well documented. Part of this geographic variation is linked to differences in population health and needs. However, some of the variation may be unwarranted and driven by factors including provider discretion, availability and distribution of resources, financing and reimbursement models.

Objectives: To develop and validate an instrument a) to assess the pSS patients experience and satisfaction along their clinical pathway including both primary care services and specialists, b) to collect comparable information in Europe to establish practice profiles in the diagnosis, management and treatment of patients with primary SjÖgren's Syndrome (pSS).

Methods: The questionnaire consists of 32 items and collects patient-reported data on: type and intensity of treatments and services received (e.g. diagnostic testing, hospitalizations, specialist visits), costs, patients' satisfaction with the care received and general information covering patients' overall health, education, ethnicity and marital status. A narrative-based medicine section is also included in the questionnaire administered to newly diagnosed patients to explore their journey to pSS diagnosis. Additionally, a short questionnaire is administered to the specialists treating the pSS patients to collect data on the organisation of their clinical centres.

Results: The pilot version of the questionnaire was administered to 164 pSS patients (mean (SD) age: 60 (12.2) yrs) from 6 clinical centres. The majority of the respondents had a primary or secondary school (59\%). Disease activity was significantly associated with frequency of rheumatologic visits and diagnostic tests $(p<0.001)$. Both the total number of specialists involved in the care other than the rheumatologist and the number of treatments received in the last 12 months before the interview varies significantly among patients and across centres $(p<0.001)$. Patients with lower education have attended on average less specialists than those with a high school or university degree $(p<0.001)$. Construct validity was supported by the questionnaire's ability to discriminate between groups with different levels of activity of the disease and socio-demographic characteristics.

Conclusions: Preliminary results confirm that the questionnaire is a valid instrument to assess and compare patterns of care for pSS patients in terms of access and utilisation of treatments and services across and within providers. Patientreported data linked with available information from clinical records will allow to measure quality of care more comprehensively and to identify best practices and opportunities for improvement, enhance care outcomes, and increase value for patients. Further analysis will be conducted in other clinical centres within the European Horizon2020 project "HarmonicSS" to verify the generalizability and additional psychometric properties of the instrument before collecting data across and within countries

Disclosure of Interest: None declared

DOI: 10.1136/annrheumdis-2018-eular.5987

\section{FRI0354}

URINE METABOLOMIC FINGERPRINT AS DIAGNOSTIC BIOMARKER FOR LUPUS NEPHRITIS

D.A. Herrera Van Oostdam ${ }^{1}$, C. Abud Mendoza ${ }^{1}$, R. Flores Ramírez ${ }^{2}$, M. Rodríguez Aguilar ${ }^{2}$, M. Pierdant Pérez ${ }^{3}$, M.U. Martínez Martínez ${ }^{1} .{ }^{1}$ Unidad Regional de Reumatología y Osteoporosis, Hospital Central "Dr. Ignacio Morones Prieto", ${ }^{2}$ Coordinación para la Innovación y Aplicación de la Ciencia y la Tecnología, Coordinación para la Innovación y Aplicación de la Ciencia y la Tecnología; ${ }^{3}$ Facultad de Medicina, Universidad Autónoma de San Luis Potosí, San Luis Potosi, Mexico

Background: Lupus nephritis (LN) represents the main prognostic factor for worsening in systemic lupus erythematosus (LES). ${ }^{1}$ The relevant classes of $L N$-due to the need of treatment- are the proliferative (III, IV, III/IV+V) and membranous (V)

Objectives: The aim of the study was to find a urinary metabolomic fingerprint to diagnose proliferative and/or membranous LN.

Methods: Cross-sectional study. Inclusion criteria: lupus patients with and with out clinical significant lupus nephritis (classes III, IV, V and mixed classes).

Urine samples were screened for metabolites using gas chromatography mass spectrometry (coupled with electronic nose). Statistical analysis: principal component analysis (PCA), and for the selection of the metabolites we used Random Forest.

Results: We included 29 lupus patients, 11 with LN. The median SLEDAI score in LN patients was of 13 vs. 3 in those without NL $(p<0.0001)$. Class IV nephritis was present in $45 \%$, mixed class in $36 \%$, and class $\mathrm{V}$ in $18 \%$. The median proteinuria of patients with NL was $1 \mathrm{~g} / \mathrm{L}$, (IQR 2.7).

The variance explained using the first two principal components was $80 \%$.

With random forest we selected, 2 nonanone, as the metabolite with the best diag nostic accuracy, (sensitivity of 0.87 and specificity of 0.93 ) of proliferative LN Obtaining the ratio of 2-bromopropane/2-nonanone, the diagnostic accuracy improved, with a positive likelihood ratio (LR) of 14 and a negative LR of 0.1 (AUC $90 \%)$

Metabolic pathways involved in LN were: methane, glycolysis, pyruvate and glyc erophospholipid pathways.

\section{Bromopropano/Nonano 2 uno}

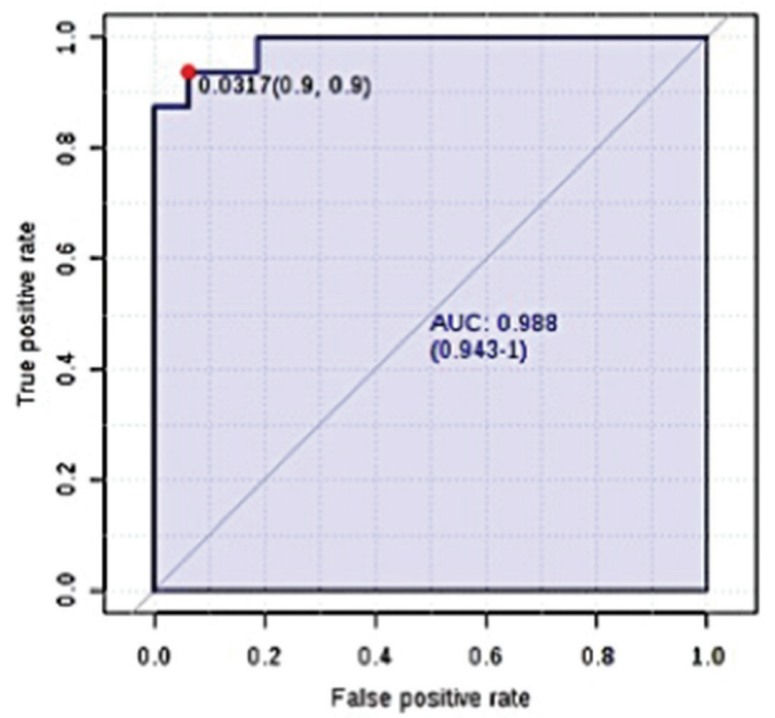

Conclusions: We identified a urinary metabolomic fingerprint that involved several metabolic pathways; 2-nonanone and the ratio of 2- bromopropane/2- nonanone had the best diagnostic accuracy in our study.

\section{REFERENCE:}

[1] Mok CC, et al. Arthritis Rheum 2013;5:2154-2160.

Disclosure of Interest: None declared

DOI: 10.1136/annrheumdis-2018-eular.7529 